\title{
Outer Membrane Vesicle Proteomics to Discover the Pathogenicity of Acinetobacter baumannii
}

\section{Vishvanath Tiwari*}

Department of Biochemistry, Central University of Rajasthan, Ajmer 305817, Rajasthan, India

Acinetobacter baumannii causes pneumonia, urinary tract infection, bacteremia, meningitis, blood stream infection and wounds of combat casualty [1,2]. The ability of Acinetobacter to adhere at abiotic surface and form biofilm helps in its survival in the harsh environmental conditions such as desiccation, nutrient deficiency and antibiotic treatment. There is rising concern about antimicrobial drug resistance among Acinetobacter baumannii since the past decade [312]. Gram negative bacteria constitutively secrete Outer Membrane Vesicles (OMVs) into the extracellular milieu that play crucial role in the delivery of virulence factors to host cells [13]. OMVs also act as intercellular communicasomes in polyspecies communities by enhancing bacterial survival, nutrient acquisition, biofilm formation, and pathogenesis $[2,13,14]$. OMVs allow enzymes/proteins to reach distant targets in a concentrated, protected, and targeted form. The gram-negative envelope also contains proteins with several important functions, such as nutrient acquisition, secretion, signaling, adherence, and protection from the environment [15]. Therefore, it is important to explore the OMVs for the better understanding of pathogenesis and virulence of Acinetobacter baumannii.

Proteomics emerged as a tool to study the proteome under diverse conditions $[4,5]$. With the advance of proteomics, a significant advancement has been made in the recent years in the field of OMV proteomics. Methods such as unlabeled (comparative coomassie stained 2D electrophoresis), labeled (DIGE, iTRAQ, ICAT, ICPL) and recently label-free quantification methods (SRM) have emerged which can be engaged in the OMV proteomics. These advancements in the proteomic methods help in the better understanding of outer membrane vesicles proteome.

Kwon et al. performed proteomics of OMVs to identify the secretion of OMVs as well as analyze the comprehensive proteome of A. baumannii-derived OMVs [16]. They reported a number of proteins such as $\beta$-lactamase, OmpA, chaperonin GroEL and virulenceassociated proteins in the OMVs of Acinetobacter [16]. Using OMVs proteomics, EF-Tu has been identified in the cell surface and OMVs of A. baumannii [17]. Mendez et al., performed proteomics of extracellular proteome such as OMVs and FSEPs (freely soluble extracellular proteins) of A. baumannii [18]. The results identified 179 proteins in the OMVs fraction and 148 proteins in the FSEPs fraction. They identified 39 OMVs proteins which are associated with pathogenesis and virulence, attachment to host cells (e.g., CsuE, CsuB, CsuA/B) and specialized secretion systems for delivery of virulence factors (e.g., $\mathrm{P}$. pilus assembly and Fil F) [18]. They also explain the role of proteins of OMVs in the oxidative stress and as defense mechanism against the macrophagic attack [19].

Cabral et al., performed proteomics of Acinetobacter cultured in three different conditions (exponential, late stationary phase and biofilms stage) and they also checked the effects of biofilm inhibitory compound (salicylate) on the biofilm formation. This multipleapproach strategy showed a unique lifestyle of A. baumannii involved in biofilms formation [20]. Similarly, high-end isoelectric point proteome analysis of Acinetobacter radioresistens reveals that 'envelope stress responses' can be induced by aromatic compounds [21-23]. Using
2D-DIGE and mass analysis, Minami et al. demonstrated that alteration in proteomic profiles of detergent-resistant membrane fractions during cold acclimation [24]. Proteomic analyses also showed that histidine metabolism have role in the biofilm formation of Acinetobacter baumannii [20]. Yun et al., performed differential quantitative proteomic analysis of outer membrane from multidrug-resistant Acinetobacter baumannii and reported that carbapenem induces the expression of resistance-nodulation-cell division transporters, protein kinases and suppress outer membrane proteins expression [19]. Carbapenem resistance of Acinetobacter baumannii has been studied using outer membrane proteomics between wild type and carbapenem resistance strain of Acinetobacter baumannii [12,16,21,22].

Reports have shown that OMV proteomics also help to develop vaccine and probiotics. Aguilera et al. identified 18 different proteins in the OMVs of Escherichia coli strain Nissle-1917. These OMV interact with the host and induce the beneficial effects on the host hence can be used as probiotics [25]. Using OMV proteomics of detergent-extracted OMVs and detergent-free extracted OMVs, Bas et al proved that the immunogenic proteins of OMV vaccines depends partially on the purification procedure and they suggested that detergent-free OMVs are preferred composition for the vaccine development against gram negative bacteria [26].

Therefore, OMVs proteomics helps to explain the role of various factors/proteins of OMVs in the pathogenesis, virulence and survival of Acinetobacter in the host. Hence, outer membrane vesicles proteomics has now emerged as a method of choice for the study of pathogenesis of disease and virulence of Acinetobacter baumannii.

\section{Acknowledgement}

I would like to thanks Monalisa Tiwari for providing help in the proofreading of the manuscript.

\section{References}

1. Bhargava N, Sharma P, Capalash N (2010) Quorum sensing in Acinetobacter: an emerging pathogen.Crit Rev Microbiol 36: 349-360.

2. Jun SH, Lee JH, Kim BR, Kim SI, Park TI, et al. (2013) Acinetobacter baumanni outer membrane vesicles elicit a potent innate immune response via membrane proteins. PLoS One.

3. Tiwari V, Moganty RR (2014) Conformational stability of OXA-51 betalactamase explains its role in carbapenem resistance of Acinetobacter baumannii. J Biomol Struct Dyn 32: 1406-1420.

*Corresponding author: Vishvanath Tiwari, Department of Biochemistry, Central University of Rajasthan, Ajmer 305817, Rajasthan, India, Tel: + 91-1463-238652; Fax: + 91-1463-238722; E-mail: vishvanath7@yahoo.co.in

Received August 24, 2014; Accepted August 27 2014; Published August 29 , 2014

Citation: Tiwari V (2014) Outer Membrane Vesicle Proteomics to Discover the Pathogenicity of Acinetobacter baumannii. J Data Mining Genomics Proteomics 5 : e116. doi:10.4172/2153-0602.1000e116

Copyright: (c) 2014 Tiwari V. This is an open-access article distributed under the terms of the Creative Commons Attribution License, which permits unrestricted use, distribution, and reproduction in any medium, provided the original author and source are credited. 
Citation: Tiwari V (2014) Outer Membrane Vesicle Proteomics to Discover the Pathogenicity of Acinetobacter baumannii. J Data Mining Genomics Proteomics 5: e116. doi:10.4172/2153-0602.1000e116

Page 2 of 2

4. Tiwari V (2014) Membrane Proteomics has emerged as a Tool to Study Carbapenem Resistance in Acinetobacter baumannii. Journal of Proteomics \& Bioinformatics

5. Tiwari $\mathrm{V}$ (2014) Differential proteomics has emerged as a tool to understand carbapenem resistance in Acinetobacter baumannii. Journal of Integrated Omics 4: 1-2.

6. Tiwari V, Moganty RR (2013) Structural studies on New Delhi Metallo-betalactamase (NDM-2) suggest old beta-lactam, penicillin to be better antibiotic for NDM-2-harbouring Acinetobacter baumanni. J Biomol Struct Dyn 31: 591-601.

7. Tiwari V (2013) Effect of Iron Availability on the Survival of CarbapenemResistant Acinetobacter baumannii: a Proteomic Approach. Journal of Proteomics \& Bioinformatics.

8. Tiwari V, Vashistt J, Kapil A, Moganty RR (2012) Comparative proteomics of inner membrane fraction from carbapenem-resistant Acinetobacter baumanni with a reference strain. PLoS One.

9. Tiwari V, Nagpal I, Subbarao N, Moganty RR (2012) In-silico modeling of a novel OXA-51 from beta-lactam-resistant Acinetobacter baumannii and its interaction with various antibiotics. J Mol Model 18: 3351-3361.

10. Tiwari V, Kapil A, Moganty RR (2012) Carbapenem-hydrolyzing oxacillinase in high resistant strains of Acinetobacter baumannii isolated from India. Microb Pathog 53: 81-86.

11. Vashist J, Tiwari V, Das R, Kapil A, Rajeswari MR (2011) Analysis of penicillinbinding proteins (PBPs) in carbapenem resistant Acinetobacter baumannii. Indian J Med Res 133: 332-338.

12. Vashist J, Tiwari V, Kapil A, Rajeswari MR (2010) Quantitative profiling and identification of outer membrane proteins of beta-lactam resistant strain of Acinetobacter baumannii. J Proteome Res 9. 1121-1128.

13. Jin JS, Kwon SO, Moon DC, Gurung M, Lee JH, et al. (2011) Acinetobacter baumannii secretes cytotoxic outer membrane protein A via outer membrane vesicles. PLoS One.

14. Jin JS, Moon DC, Choi CW, Kim SI, Kim HY, et al. (2011) A Pathogenic Potentia of Acinetobacter baumannii-Derived Membrane Vesicles. Journal of Analytical Science and Technology 2: A52-A55.

15. Kulp A, Kuehn MJ (2010) Biological functions and biogenesis of secreted bacterial outer membrane vesicles. Annu Rev Microbiol 64: 163-184.
16. Kwon SO, Gho YS, Lee JC, Kim SI (2009) Proteome analysis of outer membrane vesicles from a clinical Acinetobacter baumannii isolate. FEMS Microbiol Lett 297: 150-156.

17. Dallo SF, Zhang B, Denno J, Hong S, Tsai A, et al. (2012) Association of Acinetobacter baumannii EF-Tu with cell surface, outer membrane vesicles, and fibronectin. ScientificWorldJournal.

18. Mendez JA, Soares NC, Mateos J, Gayoso C, Rumbo C, et al. (2012 Extracellular proteome of a highly invasive multidrug-resistant clinical strain of Acinetobacter baumannii. J Proteome Res 11: 5678-5694.

19. Yun SH, Choi CW, Kwon SO, Park GW, Cho K, et al. (2011) Quantitative proteomic analysis of cell wall and plasma membrane fractions from multidrugresistant Acinetobacter baumannii. J Proteome Res 10: 459-469.

20. Cabral MP, Soares NC, Aranda J, Parreira JR, Rumbo C, et al. (2011) Proteomic and functional analyses reveal a unique lifestyle for Acinetobacter baumannii biofilms and a key role for histidine metabolism. J Proteome Res 10: 3399-3417.

21. Siroy A, Cosette P, Seyer D, Lemaitre-Guillier C, Vallenet D, et al (2006) Globa comparison of the membrane subproteomes between a multidrug-resistant Acinetobacter baumannii strain and a reference strain. J Proteome Res 5 3385-3398.

22. Marti S, Sanchez-Cespedes J, Oliveira E, Bellido D, Giralt E, et al. (2006) Proteomic analysis of a fraction enriched in cell envelope proteins of Acinetobacter baumannii. Proteomics 6: 82-87

23. Mazzoli R, Fattori P, Lamberti C, Giuffrida MG, Zapponi M, et al. (2011) High isoelectric point sub-proteome analysis of Acinetobacter radioresistens $\mathrm{S} 13$ reveals envelope stress responses induced by aromatic compounds. Mol Biosyst 7: 598-607.

24. Minami A, Fujiwara M, Furuto A, Fukao Y, Yamashita T, et al. (2009) Alterations in detergent-resistant plasma membrane microdomains in Arabidopsis thaliana during cold acclimation. Plant Cell Physiol 50: 341-359.

25. Aguilera L, Toloza L, Gimenez R, Odena A, Oliveira E, et al. (2014) Proteomic analysis of outer membrane vesicles from the probiotic strain Escherichia coli Nissle 1917. Proteomics 14: 222-229.

26. van de Waterbeemd $\mathrm{B}$, Mommen GP, Pennings JL, Eppink $\mathrm{MH}$, Wijffels $\mathrm{RH}$ et al. (2013) Quantitative proteomics reveals distinct differences in the protein content of outer membrane vesicle vaccines. J Proteome Res 12: 1898-1908. 